\title{
Males involuntarios. Para una reapropiación del concepto de «efectos perversos»
}

\section{Javier L. Cristiano}

Universidad Nacional de Córdoba. Argentina

cribau@hotmail.com

Data de recepció: febrer 2001

Data d'acceptació: setembre 2001

\section{Resumen}

Este trabajo intenta hacer, a la vez, una restricción y una ampliación del concepto de «efectos perversos» (Raymond Boudon). Restricción, porque trata de circunscribir la noción a un conjunto más específico de fenómenos que los considerados por Boudon bajo su órbita, concibiéndolos como un tipo particular de "consecuencias no intencionadas" de la acción social. Pero al mismo tiempo ampliación, porque se intenta recuperar el concepto por fuera del presupuesto de una agencia y una interacción «racional».

Palabras clave: no-intencional, agregación, racionalidad, agencia.

\section{Abstract. Unintended wrong. For a reformulation of the concept of "perverse effects»}

This paper aims at both a limiting and at the same time broadening of the "perverse effects» concept (Raymond Boudon). A limiting, since the factors encompassed by this notion are reduced to a more specific group of social facts. Thus, they are seen as a particular kind of unintended consequences of social action. At the same time its a broadening, as it tries to recuperate the "perverse effects» concept but outside of agency and "rational» interaction model.

Key words: unintended, aggregation, rationality, agency.

\section{Sumari}

I. Introducción

II. ¿Qué son los «efectos perversos»?

III. La teoría de la acción y la interacción subyacente: un ejercicio de ampliación
IV. «Efectos perversos»:

hacia una reelaboración del concepto

V. A modo de conclusión

Bibliografía 


\section{Introducción}

El concepto de «efectos perversos» fue incorporado a la sociología a mediados de los años setenta, en un texto ahora clásico de Raymond Boudon ${ }^{1}$. En ese libro, Boudon lo utiliza para referirse a diversos géneros de situaciones en que un agregado de acciones individuales produce resultados colectivos que ninguno de los participantes se había propuesto. Así definida, por tanto, la noción es virtualmente equivalente a la de «consecuencias no intencionadas de la acción social $»^{2}$, una equivalencia que Boudon reconoce y que justifica por el hecho de que, en cualquier caso, lo que está en juego es la "perversa» particularidad de la interacción social, consistente en trascender, y muchas veces burlar, las expectativas e intenciones de los agentes.

La expresión "efectos perversos» sugiere, sin embargo, un tipo específico de consecuencia involuntaria. Muchos fenómenos de interés sociológico pueden entenderse como producto involuntario de las acciones humanas, pero parece apropiado reservar la expresión «efectos perversos» para un tipo de situación en que los «resultados» tienen un carácter manifiestamente pernicioso. Este trabajo se interesa por los «efectos perversos» en este sentido más restringido. En el contexto temático de las «consecuencias no intencionadas de la acción social», deseamos concentrar la atención en un tipo específico de consecuencias: las que puedan merecer el adjetivo de "perversas».

Interesarse por este problema es ubicarse al costado de dos caminos muy transitados en la teoría sociológica. Por un lado, el que consiste en prestar atención a fenómenos sociales «indeseables», pero intencionales y deliberados. Las teorías «conspirativas» del poder son el ejemplo sobresaliente (aunque no el único). Por otro, el que distingue a cierto género de teoría "sistémica», para el cual las totalidades sociales trascienden las intenciones de los individuos, pero no para provocar perjuicios, sino beneficios en términos de armonía, estabilidad, evitación de conflictos y orden social. En un caso, la «perversidad» es atribuida a unos concretos agentes cuya intención (o mala intención) funciona como explanans. En el otro, la capacidad autoorganizadora de totalidades abstractas se encarga de coordinar y dirigir las consecuencias, haciendo innecesaria la preocupación por las «intenciones» $\mathrm{o}$ «deseos» de los agentes, y tornando secundarios los problemas de lo inesperado y de lo indeseable. Ninguno de estos puntos de vista es lógicamente incompatible con la tematización de «efectos perversos», pero es evidente que predisponen escasamente para su atención y tratamiento.

Ahora bien; el concepto de «efectos perversos», tal como Boudon lo utiliza, está enlazado al presupuesto del carácter «racional» de los agentes sociales.

1. Boudon, R. (1982). En determinados contextos, el autor habla también de «efectos de composición", «efectos emergentes» o "efectos de agregación».

2. Merton (1980). Un repaso de la historia del problema se encuentra en Hayek (1978) y en Lamo de Espinosa (1990). Para tratamientos específicos del mismo pueden consultarse también Merton (1989), Elias (1982), Hirschman (1994), Ramos (1993), Giddens (1984) y Elster (1996). 
Más precisamente, la elucidación de efectos perversos se apoya para Boudon en la suposición de actores racionales, sino "perfectos», al menos de "racionalidad compleja ${ }^{3}$. Esto lo coloca en una amplia e importante estela de autores que, partiendo de opciones parecidas, se han ocupado de dilemas de decisión, estructuras de interacción, problemas de acción colectiva y temáticas afines ${ }^{4}$. Nuestro trabajo surge de lo que es ya un lugar común respecto de esta perspectiva: el convencimiento de los límites del modelo de la racionalidad (sea fuerte o débil) como paradigma explicativo de la acción y la interacción social. Se pretende aquí, en efecto, analizar la posibilidad de una extensión del concepto de "efectos perversos», de manera tal que sea aplicable al análisis social sin necesidad de ser sostenido por un concepto de agencia como el que expresa el punto de vista «racionalista».

Lo primero, por lo tanto, es intentar una definición algo más precisa de «efectos perversos», como subtipo de "consecuencias no intencionadas de la acción social» (apartado II). Se tratará de una definición preliminar, que será retomada y reelaborada al final del trabajo. En segundo lugar (apartado III), debemos pensar una extensión del modelo de agencia e interacción, de modo de ir más allá del paradigma de la racionalidad. En esta tarea nos valdremos de algunas ideas de Anthony Giddens, no para asumirlas en su totalidad (no se pretende discutir aquí su validez), sino como parte de un ejercicio de ampliación del concepto de agencia e interacción. Tercero, habrá que retornar, con esta nueva dotación conceptual, a la noción de «efectos perversos» (apartado IV). Propondremos no una definición, sino una caracterización de la noción de «efectos perversos» que se apoye, ahora, en el más amplio y matizado concepto de agencia e interacción proporcionado por la «teoría de la estructuración».

\section{II. ¿Qué son los «efectos perversos»?}

¿Qué tipos de consecuencias no intencionadas son aquéllas que podemos llamar "efectos perversos»? Para comenzar a responder esta pregunta, vamos a valernos de una taxonomía propuesta por Patrick Baert (1991), quien distingue las siguientes variables para analizar, en general, la naturaleza de las «consecuencias no intencionadas» de la acción social:

a) A quién se refiere el efecto de las acciones: al actor/es cuya acción produce el efecto, a otro/s actor/es, o a colectivos más amplios (ya sean efectos «sistémicos» o efectos «de agregación», que involucran a un grupo de individuos).

3. Boudon habla de "racionalidad compleja» para diferenciar su punto de vista de los modelos "puros» de racionalidad, como el de las "acciones lógicas» de Pareto. Esto lo distancia al mismo tiempo de las versiones radicales del «imperialismo económico» (por ejemplo Becker, 1980). Sin embargo, el supuesto central de la "racionalidad compleja» continúa siendo que los agentes obran persiguiendo fines y estimando de algún modo los medios para alcanzarlos. Un desarrollo más detenido de la cuestión se encuentra en Boudon (1986).

4. Consúltese, por ejemplo, la compilación de Fernando Aguiar (1991). 
b) Valor atribuido al efecto desde la perspectiva del/de los actor/es involucrado/s en la acción: efectos deseables, indeseables o neutrales.

c) Relación con la intención inicial: efectos que cumplen, frustran o que ni cumplen ni frustran la/s intención/ciones inicial/es.

d) Relación con la cognoscibilidad y conciencia del/de los actor/es involucrados: efectos no esperados, o esperados pero no ocurridos.

e) Aspectos temporales: efectos sincrónicos (simultáneos a la realización de $\mathrm{la} / \mathrm{s}$ acción/ciones involucrada/s), o efectos diacrónicos (posteriores a la/s acción/ciones involucrada/s).

En primer lugar, un efecto puede ser llamado "perverso» con independencia del nivel sobre el cual se produzca (dimensión «a»). La "perversidad» puede afectar a los actores que producen el efecto con su acción, a otros actores o, en una consideración teórica más amplia, al «sistema» o a una totalidad definida teóricamente de algún modo. En relación con la segunda dimensión, los efectos perversos se refieren a consecuencias que, desde el punto de vista de los actores, pueden ser indeseables o neutrales (si se producen sobre otros individuos o sobre totalidades sistémicas). Respecto de la tercera dimensión, los efectos perversos son aquéllos que frustran la intención inicial de los actores o, de lo contrario, efectos neutrales para esos actores que, sin embargo, pueden ser calificados como "perversos» para otros actores o para alguna totalidad. De acuerdo con la cuarta dimensión, los efectos perversos son, naturalmente, los no esperados por los actores (aunque puedan ser esperados por otros actores que no participan en las acciones que producen el efecto). La quinta dimensión no tiene mayor relevancia para la definición: los efectos perversos pueden ser tanto sincrónicos como diacrónicos.

Con todo lo que dejan todavía por dilucidar, estas precisiones permiten de momento clarificar algo esencial: que unas consecuencias específicas de acciones sociales pueden ser definidas como "perversas» tomando en consideración (1) una referencia a «dimensiones subjetivas» de los actores sociales (intenciones y deseos, ya sea de los que producen las consecuencias o de otros actores); (2) unos presupuestos teóricos desde los cuales afirmar «bondades» o "falencias» en el funcionamiento de algún tipo de totalidad societaria, y (3) una valoración moral del analista. Así, por ejemplo, el caso prototípico de «efecto perverso» que es la inflación, puede ser calificado de tal tomando en consideración los deseos de - presumiblemente - la mayoría de los individuos (seguramente no de todos), ciertos principios de equidad, justicia y bienestar (que, también presumiblemente, el analista comparte con los actores), y una visión teórica desde la cual definir, por ejemplo, lo que es una «economía sana» o «insana» (las teorías antimonetaristas niegan, por ejemplo, que la inflación sea necesariamente un indicador negativo). En cambio, uno de los casos que analiza el propio Boudon (a saber: que el incremento de las oportunidades educativas ha producido la inesperada y "perversa» consecuencia de aumentar los costos de la educación, sin aumentar la movilidad social), implica una presuposición acerca de la subjetividad de los actores (que es deseable para algunos e indeseable para otros, la 
movilidad social), y tal vez un conjunto de valoraciones del propio Boudon (ligadas a la deseabilidad de la movilidad social y de la igualdad de oportunidades), pero no una consideración sistémica (no sabemos si el efecto producido sobre la estructura social es «favorable» o "desfavorable» para el sistema social).

Por supuesto, estos tres parámetros (subjetividad de los actores, teoría y valores del analista) suelen aparecer complejamente entrelazados; y por cierto también que cada uno de ellos acarrea un amplio espectro de problemas teóricos y filosóficos ${ }^{5}$. De momento, la formulación es suficiente para encarar el análisis que sigue. Se concibe, por tanto, a los «efectos perversos» de acciones sociales, como aquellas consecuencias que no han estado incluidas entre las metas de las acciones que las producen y que, a la luz de intenciones/deseos de actores, necesidades teóricamente establecidas de funcionamiento societario, o valores morales determinados, provocan algún tipo de perjuicio a actores y/o sistemas.

\section{La teoría de la acción y la interacción subyacente: un ejercicio de ampliación}

La definición anterior deja claro que no sólo la identificación de «efectos perversos», sino también su explicación (es decir: de qué modo y por qué ellos ocurren) suponen una conceptualización de la acción y la interacción social. Aunque sin ánimo de exhaustividad, este apartado se dedica a esta cuestión. Comencemos analizando brevemente tres ejemplos de «efectos perversos» que Boudon trata en su libro.

Oportunidades educativas y movilidad social. En sus propias investigaciones empíricas, Boudon constata que, contrariamente a las expectativas de sus impulsores, el crecimiento de las oportunidades educativas no va acompañado de una igualación social; al contrario, conlleva mayores desigualdades sociales, además de significar para todos los miembros de la sociedad un incremento en el «costo del estatus»: para estar en un mismo lugar relativo de la jerarquía social, hace falta una inversión educativa cada vez mayor.

Costos y beneficios de la acción colectiva. La estructura de la interacción es aquí la del dilema del prisionero ${ }^{6}$. De acuerdo con el conocido análisis de Mancur Olson (Olson, 1992), en una situación de acción colectiva se da el siguiente dilema: es beneficioso para todos que todos cooperen (que todos participen, por ejemplo, en una protesta), pero el interés de cada uno, individualmente, consiste en no participar y beneficiarse de la participación de los otros. Obrando de acuerdo con su interés individual, la suma de las acciones individuales con-

5. Brevemente, y a título ilustrativo: los problemas de la subjetividad y la «comprensión del sentido» (el primer parámetro); los problemas de la validez de teorías alternativas y, en particular, de las concepciones de totalidad social (el segundo), y los problemas de la participación de los valores en la producción del conocimiento sociológico (el tercero).

6. Sobre el «dilema del prisionero» — de evidente interés para la problemática de los «efectos perversos»—, pueden verse Poundstone (1995) o Axelrod (1986). 
duce al aborto de la acción colectiva, y la consiguiente permanencia en la situación problema.

Segregación involuntaria. Boudon toma el análisis de Tomas Schelling (Schelling, 1971), que demuestra el modo como una pauta de segregación espacial puede producirse sin que nadie de los involucrados se haya propuesto producirla. Es suficiente con que cada uno tienda a localizarse físicamente (por ejemplo, alquilar una vivienda) en lugares en los que sus pares (raciales, por ejemplo) sean mayoría o, al menos, no sean minoría. El resultado será, inevitablemente, la formación de guetos.

Estos ejemplos — presentados aquí, hay que subrayarlo, de una manera excesivamente simplificada - son suficientes para mostrar el tipo de situaciones que Boudon tiene en mente cuando habla de «efectos perversos». En primer lugar, las acciones involucradas son de un tipo muy específico. Son acciones en las cuales es razonable suponer la existencia de una deliberación consciente, previa a la acción, en la medida en que las situaciones salen de la rutina e implican, a los ojos del agente, la puesta en juego de costos y beneficios potenciales que están más allá de lo habitual. En otras palabras, acciones en las que resulta claro para el actor que es su propio comportamiento lo que habrá de acarrear unas consecuencias que serán para él beneficiosas o perjudiciales. Decidir participar o no en una cacería no convencional, participar o no en una huelga, vivir en un lugar o en otro de la ciudad, educarse en uno u otro colegio, o suspender el proceso educativo en uno u otro nivel, son acciones evidentemente no "rutinarias», aún cuando en ciertas condiciones puedan darse con recurrencia mayor.

En segundo lugar, y por lo que respecta a los «resultados» o «efectos», se trata también de unos casos particulares. La cacería rousseauniana deriva en la posibilidad de una alimentación mejor y riesgosa, o una habitual y segura. En el ejemplo de la educación, lo que está en juego es el costo de la educación en términos de tiempo y dinero, y la posibilidad de ascenso en la escala social, que puede asociarse a variables como el ingreso salarial. Los casos de acción colectiva pueden ser muy variados, pero se trata en general de acciones destinadas a lograr beneficios económicos (aumentos salariales, reducción de impuestos, etc.) o políticos (acceso a ciertas instancias de poder o, al menos, reformulación de las relaciones de fuerza con instituciones o grupos). En el ejemplo tomado de Schelling, el resultado de las acciones agregadas es un mayor o menor aislamiento espacial de los grupos étnicos. Todos estos «efectos» consisten en «recompensas» o "pérdidas», todas ellas de carácter relativamente concreto, e incluso en ocaciones fáciles de mensurar, además de ser más o menos fácilmente valorables como positivos («beneficios») o negativos ("pérdidas»). Pero está claro que no todos los «efectos» de acciones individuales tienen estas características ${ }^{7}$.

7. En Boudon (1981) se discuten muchos otros ejemplos de «efectos perversos» o «de composición». Así, por ejemplo, el conocido estudio de Merton sobre la discriminación racial 
Como quedó dicho, no puede adjudicársele a Boudon ninguna clase de «imperialismo». Pero si queremos avanzar hacia una conceptualización más abarcativa del concepto de "efectos perversos», tenemos que asumir esta circunscripción como una limitación. Y esto significa básicamente lo siguiente: puesto que (de acuerdo con la definición preliminar de II) también puede hablarse de «efectos perversos» en casos en los cuales las acciones y los efectos involucrados no necesariamente tienen las particularidades señaladas, necesitamos anclar la idea de «efectos perversos» en una conceptualización más amplia de la acción y la interacción. Las formas de hacerlo que están disponibles en la teoría sociológica son diversas; hemos optado aquí por recurrir a la teoría de la estructuración y, específicamente, a algunos de los elementos del modelo de agencia y de interacción elaborado por Giddens ${ }^{8}$.

Siguiendo a Giddens, lo primero que hay que asumir es que esa acción en la cual el agente define conscientemente una meta, sopesa estrategias y resultados posibles en función de sus conocimientos, y toma luego una decisión sobre el curso de acción apropiado, es sólo el caso extremo de un abanico de posibilidades mucho más amplio. Y, sobre todo, hay que asumir que no hay razón alguna para asignar a priori a ese caso un predominio empírico. Aceptado esto, se hace visible la necesidad de repensar las categorías — "conciencia», «intención», «razón», etc.— que Boudon invoca para dar cuenta de la vida subjetiva que acompaña a las acciones humanas.

Para empezar, la "conciencia» que acompaña al acto no tiene necesariamente la forma de un saber inmediatamente accesible para el actor. No es inevitable que el agente sea capaz de poner en palabras los objetivos que persigue con su acción, y las razones por las cuales ella resulta adecuada a esos objetivos. "La mayor parte de la corriente de acción que constituye la conducta cotidiana es prerreflexiva» (Giddens, 1987: 78), lo cual significa que la acción social no es una sucesión de actos precedidos por deliberación y decisión consciente, sino más bien un continuo en el que se entrelazan la inercia de la rutina, la vaga referencia a "proyectos» que trascienden temporal y espacialmente al acto presente y a veces — pero sólo a veces - el ajuste deliberado de la acción a unas metas concretas. Mejor que hablar de "conciencia», en el sentido de la

en los sindicatos norteamericanos en la entreguerra, la «ley de la tasa decreciente de ganancia», de Marx, o las sucesivas decisiones de Alemania y los aliados en el período previo a la Segunda Guerra Mundial. Con pocas excepciones, lo dicho vale también, en lineas generales, para estos análisis.

8. Para un ejercicio tentativo como éste, la obra de Giddens ofrece la ventaja de ser un intento de síntesis y superación de un número importante de tradiciones precedentes. En tal sentido, se puede considerar que en sus escritos se reúnen aportes diversos que de otra manera habría que reconstruir por cuenta propia. Pero debe quedar claro que habría otras maneras de realizar esta tarea (considerando, por ejemplo, las obras también sintetizadoras y superadoras de Bourdieu o Habermas) y que, además, llevar hasta el límite este intento supondría discutir la pertinencia de la síntesis que propone Giddens. Por obvias razones no lo hacemos aquí. Hemos tomado en consideración, para lo que sigue, a Giddens (1987 y 1995). 
puesta en juego, a sabiendas y deliberada, de unos conocimientos sobre el mundo, es hablar de un "registro reflexivo" de la acción, en el sentido de que toda acción es acompañada de algún tipo de registro cognitivo, pero ese registro no es un conocimiento necesariamente consciente.

Esta matización nos conduce a un primer ajuste importante respecto del modelo de la racionalidad, y es que las acciones que se entrelazan para producir un «resultado" (por ejemplo, un "efecto perverso»), no sólo no son necesariamente conscientes y racionales, sino que no tienen por qué ser acciones de un mismo tipo. En una interacción pueden trabar vínculo acciones racionales con acciones rutinarias y «sin motivos», acciones alimentadas por componentes de conciencia práctica, con los más diversos «proyectos», en las más variadas proyecciones temporales, etc.

Dejemos de lado los problemas de la vida subjetiva de los actores, y pasemos a otra particularidad del modelo racional: su relativa desatención de las posiciones sociales ocupadas por los actores y, más precisamente, del modo como esa posición predefine sus acciones y, sobre todo, su potencial influencia en el curso de la interacción y de sus resultados. Tal como lo ilustran los ejemplos del comienzo de este apartado, el tipo de análisis de la acción racional tiende a presuponer un agente que, o bien se desempeña en una suerte de vacío social, a solas con sus objetivos, conocimientos y decisiones, o bien, estando socialmente situado, parece detentar siempre una muy poderosa capacidad para desmarcarse de la situación y de sus condicionamientos.

Como ha sostenido Giddens, el papel de las estructuras en la configuración de la acción tiene un carácter dual, a la vez de constreñimiento/limitación, y de habilitación/posibilidad. La estructura social está allí no sólo para impedir, reprimir y sofrenar, sino también para posibilitar y permitir. Funciona como el lenguaje, que abre un enorme campo de posibilidades de expresión, pero al mismo tiempo coloca límites a lo que puede ser pensado y enunciado. Pero no hay acción — esto es lo importante-, por reflexiva que sea, que no se nutra de los recursos y se enrole en los determinantes de la estructura. En este específico sentido no hay "afuera» respecto del orden social y de las estructuras.

Reconocer esta gravitación de la estructura es un paso decisivo en la superación del modelo racional. Porque, aún manteniéndose en los límites de ese modelo, es evidente que las limitaciones/habilitaciones de la estructura son una variable central para entender fenómenos como la posesión de información sobre el entorno, la disponibilidad de «medios» de acción o, menos obviamente, la definición de metas u objetivos de la acción. Así, por ejemplo, si queremos entender el resultado efectivo de una situación de «dilema del prisionero", tendremos que tomar en consideración el espacio social que ocupan los agentes, al menos para entender por qué, por ejemplo, el carácter dilemático de la situación resulta más claro para algunos agentes que para otros (una diferencia que, a su vez, tendrá sus consecuencias en el agregado). En resumen, asumir la importancia de la estructura obliga a llevar el análisis más allá de la simulación ideal típica, en una suerte de vacío social, de intercambios racionalmente motivados. 
Pero los argumentos de Giddens aportan algo más en este punto. Como se sabe, la teoría de la estructuración define a la estructura social como el sustrato de «reglas» $\mathrm{y}$ "recursos» que hacen posible la acción y la interacción. Estos componentes de la estructura se manifiestan en la muy concreta presencia de normas, poder y significados. Así como la abstracción de la lengua se materializa en concretos actos de habla y relaciones de comunicación, la estructura social se actualiza en la interacción como presencia e influjo de normas y regulaciones, circulación de sentidos y significados y desigualdades de poder entre los participantes.

Por evidente que pueda parecer la presencia de estos componentes, ellos no tienen un lugar importante en los análisis de Boudon. En algunos casos simplemente no aparecen como variables, y en otros, aunque estén presentes, se les asigna un estatuto ontológico relativamente simplificado y un valor explicativo secundario. Las normas y el poder se reducen a medios o límites de la acción, y las significaciones, a posesión de información. Si asumimos en su real significado la propuesta de Giddens, tenemos que sacar en cambio dos conclusiones bien distintas. Primero, que las relaciones de poder que se producen entre los partícipes de una interacción no son un elemento secundario, sino una variable explicativa fundamental no sólo de las acciones individuales, sino también —y lo que es más importante para nosotros- de los resultados agregados de la interacción. La confluencia de un conjunto de actores en una situación de interacción no los coloca de por sí en igualdad de condiciones para gravitar en los resultados agregados. Y ello no sólo en el plano más evidente del uso consciente del poder (manipulación, control, etc.), sino también en el plano de lo «no deliberado» o «no buscado»: la posesión o desposesión de «recursos» dota a ciertas acciones de un mayor poder de influencia sobre las resultantes, aún cuando el agente, conscientemente, no desee ejercer esa influencia. En este sentido, es de la mayor importancia la amplia concepción del poder que propone Giddens, no como propiedad de algunas acciones en particular (por ejemplo, las de los grupos «dominantes»), sino como un componente intrínseco de toda acción, entendida como «intervención causal corpórea» (Giddens, 1987: 77) en el proceso en marcha de los eventos externos. Pero esa intervención causal es función de una posesión de recursos que, salvo excepciones, es marcadamente desigual.

La segunda conclusión es que la producción y circulación de significados es también una variable ineludible para entender el modo como unas acciones agregadas provocan «resultados». Dicho de otra manera, los aspectos «inmateriales» de la acción y la interacción no se reducen a la utilización de «información» por parte de los agentes, sino que incluyen un complejo entramado de textos y discursos que no son externos a la interacción, sino que la constituyen con el mismo derecho que la «materialidad" de los comportamientos (la «intervención causal corpórea»). La circulación de sentido es constitutiva 
de los procesos de producción de consecuencias, y no puede por tanto ser excluida del análisis.

Esta segunda conclusión nos lleva a otra cuestión, que es la de la naturaleza de los «resultados» o los «efectos agregados». Como vimos al comienzo de este apartado, los análisis de Boudon tienden a centrar la atención en un tipo muy específico de consecuencias, particularmente concretas e incluso mensurables. La presencia de los fenómenos simbólicos en la interacción llama la atención sobre el hecho de que también en ese plano, el del «sentido», se producen "consecuencias» $\mathrm{o}$ "resultados agregados». En este punto no es necesario apelar a la pormenorizada conceptualización giddensiana de lo que ocurre «hacia fuera» de las acciones y como "consecuencia» de ellas. Es por demás evidente que el terreno desborda el limitado ámbito de las «recompensas» de los agentes, y se desplaza hacia consideraciones sociológicas más complejas. Sin entrar en ellas, tenemos que asumir por lo menos lo siguiente: 1) los «resultados» o «efectos agregados» de las acciones humanas tienen una naturaleza plural: van desde efectos materiales relativamente fáciles de percibir por los agentes y por el analista (la producción y distribución de bienes, por ejemplo), hasta la menos perceptible y casi siempre mucho más lenta constitución y reconstitución de órdenes simbólicos (representaciones, lenguaje, creencias, etc.), pasando por la reproducción y reconstitución de lógicas sociales abstractas, como las que designan las nociones de "estructura social», «institución» o «sistema», y 2) la producción de consecuencias no buscadas y "efectos perversos» no tiene por qué darse, para el caso de unas específicas acciones, en uno de estos planos en forma exclusiva, de modo que la concentración del análisis en uno de ellos es básicamente una decisión del analista. En el decurso virtualmente infinito de la producción de consecuencias, hay una decisión del analista para delimitar lo que es digno de consideración.

Acompañada o no por Giddens, esta revisión de la teoría de la acción podría continuar. Para nuestros objetivos presentes, sin embargo, es más que suficiente con lo dicho. Sinteticemos:

1. La vida subjetiva que acompaña a las acciones, y que parcialmente las explica, no sólo no se ajusta necesariamente al modelo de la racionalidad (sea pura o "compleja»), sino que tampoco es analizable, necesariamente, en términos de fijación consciente de metas u objetivos («intenciones»), y de conciencia discursiva plena de esas metas y objetivos.

2. No obstante, todos los «tipos» de acciones tienen "poder», en el sentido de estar en condiciones de intervenir causalmente en el desarrollo de los eventos sociales externos. Todas están potencialmente en condiciones de producir consecuencias, o al menos de participar en la producción de consecuencias. Por lo tanto, las combinaciones posibles de tipos de acción capaces de producir "efectos perversos» son muy variadas, siendo la que privilegia el modelo de la racionalidad (combinación de acciones racionales y/o intencionales) sólo una de las posibles.

3. El vínculo de los agentes con la «estructura social» es parte de la explicación de sus acciones, y por lo tanto es también una variable explicativa de 
la producción de «consecuencias» (entre ellas, los «efectos perversos»). De modo que para entender por qué se ha producido una determinada resultante colectiva, la atención al vínculo entre agentes, por un lado, y reglas/recursos, por otro, resulta indispensable.

4. En particular, la naturaleza y el volumen de los recursos de que disponen los agentes es una variable operante en la fijación del sentido y la magnitud de su influencia individual en la producción de «efectos perversos».

5. La producción de «efectos perversos» no es necesariamente un resultado de acciones adaptativas basadas en la posesión de «información». La producción y circulación de sentidos (representaciones, creencias, etc.) es parte constitutiva de las dinámicas de interacción que conducen a resultantes, sentidos que no pueden reducirse a la noción de «información», «datos» o afines.

6. Un conjunto de acciones agregadas pueden producir resultantes de naturaleza muy distinta, siendo sólo un tipo el que puede comprenderse y ser vivido como distribución de «recompensas». Los «efectos perversos» sobre representaciones o creencias, o sobre la estructura social, son ejemplos de otros tipos posibles.

7. Las consecuencias de las acciones humanas (incluidos sus «efectos perversos») son potencialmente infinitas en tiempo y espacio. Por lo tanto, el interés por unos resultados particulares es una decisión del analista, consistente en fijar la atención en un punto específico del tiempo.

\section{IV. «Efectos perversos»: hacia una reelaboración del concepto}

La primera impresión que resulta de estas afirmaciones es la notoria complejización que introducen al problema de los «efectos perversos». Y aunque la complejidad en sí misma no es ni buena ni mala, la pregunta que suscita en este caso es hasta qué punto puede ser re-asimilada por el concepto de «efectos perversos». Dicho en otras palabras, hay que preguntarse si la noción misma de «efectos perversos» no se vuelve inocua cuando, despojándola de su sustento en el paradigma de la racionalidad, multiplica sus dimensiones y crece en dispersión y complejidad.

En nuestra opinión, que la nueva noción de «efectos perversos» se revele como apropiada o que, por el contrario, deba ser descompuesta en sub-categorías, o reemplazada por otras nociones, es algo que sólo puede resolverse analizando las situaciones empíricas en las que podría prestar utilidad. Ésta es precisamente la tarea que ahora se impone, aunque sólo vamos a realizarla en forma indicativa e ilustrativa. Nos valdremos de la investigación de un caso de «efecto perverso» ${ }^{10}$, para

10. Se trata de una investigación llevada a cabo por Mark Fishman (1979) en los Estados Unidos. El trabajo se inscribe en el marco conceptual de la llamada Teoría de "Newsmaking», que pretende elucidar los mecanismos internos de la producción de información en el marco de organizaciones periodísticas orientadas como empresas lucrativas. De la brillante indagación de Fishman, tomamos en consideración sólo un fragmento («La producción de una ola de crímenes», ibídem, p. 9 y s.). 
intentar luego formalizar los elementos que conforman el proceso y que nos acercan (aunque sólo eso: nos acercan) a los posibles componentes de la nueva noción.

Antes de entrar en ese análisis, hay que señalar que la síntesis del apartado anterior pone en evidencia que el concepto de "efectos perversos» no puede designar solamente - como lo ha hecho en el primer apartado- un tipo entre otros de "consecuencias no intencionadas». En tal caso, el problema de explicar «efectos perversos» es virtualmente equivalente al de explicar consecuencias no intencionales en general, una equivalencia que hay que evitar si esperamos que el concepto no sólo sirva para identificar un tipo de fenómeno, sino también para explicarlo. Es necesario, en suma, repensar el concepto de «efectos perversos» para que no sólo designe resultados en algún sentido «negativos» o «indeseables» (aunque esta es una parte de la definición), sino también algunas propiedades específicas de los procesos de interacción que conduce a ellos. Veamos entonces el caso empírico, y retornemos luego a la discusión teórica.

La creación periodística de una ola de crímenes. El trabajo de Fishman intenta elucidar lo que a primera vista se presenta como una deliberada «tergiversación de la realidad» por parte de un grupo de medios de prensa norteamericanos. Después de haber informado durante varias semanas de una sucesión de ataques violentos contra ancianos, en lo que virtualmente se había convertido en una ola delictiva contra ese grupo, los registros estadísticos mostraron que no había existido durante ese tiempo ningún incremento de tales delitos, e incluso había tenido lugar una leve disminución. ¿Qué había sucedido? Según la reconstrucción de Fishman, todo comenzó cuando el jefe de Redacción de uno de los periódicos de la ciudad tuvo la idea de publicar una serie de artículos sobre delitos contra ancianos, una decisión tomada como tantas otras del mismo tipo. Encargó entonces la tarea a uno de los cronistas, el cual trabó contacto con una dependencia policial dedicada especialmente a la protección de ancianos. Familiarizándose con ese ámbito, el cronista supo de las quejas de los miembros de esa dependencia que (al igual que, presumiblemente, muchas otras) se sentían agobiados por la escasez de recursos para la tarea encomendada. Pero además, los miembros de la dependencia policial mantuvieron intensamente al tanto al cronista de los nuevos casos que se iban sucediendo, lo cual redundó en una presencia inusitada del tema en las páginas del periódico. Dado el valor periodístico que usualmente asignan los medios a acontecimientos de esta naturaleza (el daño a personas indefensas es el tipo de hechos que suscitan inmediata indignación colectiva), la llama encendida por el periódico no tardó en prender en los otros medios, que pronto se hicieron eco de las notificaciones de la unidad policial, dando virtualmente inicio a una «ola» de ataques contra ancianos.

Un momento clave del proceso tuvo lugar cuando, en una conversación ocasional, los policías mostraron descontento ante un cronista por la facilidad con que las autoridades judiciales solían dejar en libertad a los menores apresados. El cronista pidió entonces datos de casos concretos, y recibió uno: el de 
un joven negro que golpeó y robó a una anciana, para evitar luego la prisión por el pago de una fianza. En este punto entra en acción un congresista de Estado, que actuaba además en una comisión dedicada a examinar la actuación de la justicia en casos de delincuencia juvenil. Luego de leer la información en el periódico, el congresista indagó archivos y encontró que el joven delincuente tenía un antecedente de homicidio. Se puso en contacto con varios medios periodísticos, que dieron gran publicidad a la primicia. $\mathrm{Al}$ ver la intensidad con que se ocupaban los medios de él, el joven delincuente se fugó de la ciudad (no obstante la fianza), acontecimiento que irritó más aún a la opinión pública y que fue ampliamente destacado en las siguientes coberturas periodísticas. Fue entonces que el alcalde mismo, ante la presión pública sobre el tema, llamó a conferencia de prensa para anunciar que «declaraba la guerra» a los crímenes contra ancianos. Y así lo hizo. Entre otras medidas, amplió las atribuciones de la unidad policial especial, permitiendo a sus miembros actuar en ropas de civil. Esto hizo posible que tomaran contacto con una gran cantidad de hechos que, en el funcionamiento habitual de la unidad, sencillamente no eran conocidos. Otra medida fue declarar hechos de interés los ataques contra ancianos, lo cual significó, en el interior de la dependencia policial, su inclusión dentro de las cuestiones de competencia de la central. Puesto que la central emitía un informe diario para la prensa detallando los hechos delictivos ocurridos bajo su órbita, los ataques contra ancianos pasaron a tener un lugar destacado en el parte policial cotidiano.

Comencemos examinando la naturaleza de las acciones que, en conjunto, desencadenan el proceso. Tenemos al menos cuatro grupos de protagonistas: los periodistas, los policías, los funcionarios y el público de las noticias. Respecto de los primeros es posible reconocer, en primer lugar, un conjunto de acciones abiertamente instrumentales, justificadas por fines y objetivos característicos de la actividad: la búsqueda de rating, el cuidado en la presentación «objetiva» $\mathrm{y}$ «fundada» de la información, la competencia con los pares, la asunción instrumental de objetivos y órdenes de los superiores, etc. Sin embargo - y en esto la investigación completa de Fishman es un verdadero muestrario de ejemplos- muchos de los comportamientos de los periodistas no están en absoluto mediados por deliberación consciente, ni son por tanto asimilables al género "conducta propositiva». En las pequeñas decisiones cotidianas de su trabajo (por ejemplo, la decisión del cronista de solicitar a los policías ejemplos concretos de sus quejas), los periodistas, al igual que otros profesionales, ponen en juego un complejo arsenal de saberes prácticos, difícilmente accesibles a su "conciencia discursiva», los cuales no obstante orientan su comportamiento. Es posible pensar que sin esos elementos más o menos espontáneos y ajenos a la deliberación consciente, muchas de las acciones que fueron tejiendo el proceso no hubiesen tenido lugar.

Consideraciones parecidas pueden formularse respecto de las muchas acciones de miembros de los otros tres grupos: desde la (seguramente) deliberada instrumentalidad del alcalde y su «declaración de guerra» a la ola criminal, hasta el rutinario y sombrío "cumplimiento del deber» por parte de los fun- 
cionarios policiales de menor rango (por ejemplo, quienes transcriben las notificaciones para la prensa), pasando por las infinitas motivaciones subjetivas de los miles de televidentes, oyentes y lectores que, con los más variados intereses y expectativas, se posicionan frente a los productos informativos.

La descripción es elocuente, por otra parte, respecto de la importancia de los recursos de cada participante en la producción del resultado agregado. En el interior del periódico que inicia el proceso, el jefe de Redacción es quien tiene las facultades para poner en marcha el crescendo de atención sobre el tema, aún cuando no se lo haya propuesto. Las cruciales acciones del congresista de Estado y del alcalde — que ponen en juego poderosos recursos de coordinación- se destacan naturalmente por encima de, por ejemplo, los miembros individuales del público, cuyo aporte a la resultante es, individualmente considerado, ínfimo. Por otra parte, es de suponer que el periódico que inicia el proceso tiene, a los ojos de sus competidores, suficiente "poder» (medible en número de lectores, anunciantes, prestigio, etc.), como para que se monten sobre los acontecimientos de que él informa.

$\mathrm{El}$ «efecto perverso» que está en juego se produce sobre el sentido (en particular: es una creencia aceptada más o menos masivamente), lo cual quiere decir que difícilmente pueda ser evaluado, como tal, en términos de "pérdidas» o «recompensas» para los agentes intervinientes. Además, incluye al interior mismo de su producción la circulación del sentido: los propios periodistas creen en la existencia de la ola criminal, y también los funcionarios, creencia que alimentan y expanden, pero que además explica sus acciones.

Ahora bien, todos estos elementos podrían estar igualmente presentes en la producción de resultados que no fuesen «efectos perversos». Por ejemplo, podríamos explicar de una forma muy parecida las razones por las cuales se suscita una creencia pública cualquiera, y no necesariamente una «falsa» $\mathrm{O}$ "perniciosa». El caso analizado por Fishman, sin embargo, tiene al menos otras cinco características, que hacen de él un fenómeno particularmente "perverso», y no sólo por la naturaleza del resultado alcanzado.

En primer lugar, la invención de la ola criminal es exactamente el anverso de lo que, presumiblemente, los periodistas se proponen en términos de exigencia e ideal profesional. Proponiéndose suministrar a sus audiencias notificaciones en algún sentido "veraces», suministran en cambio una gigantesca «mentira», una notificación conjunta radicalmente falsa de los acontecimientos. La creencia en la ola de crímenes no es cualquier resultado negativo, sino uno que está en conflicto manifiesto con pretensiones de (al menos) una parte de los agentes involucrados. No sólo se alcanza un efecto negativo, sino que se lo alcanza pretendiendo lograr (entre otras cosas) precisamente lo contrario.

La segunda característica destacable es que, si bien todos los participantes «aportan» algo a la producción conjunta del efecto, ninguno de ellos está en condiciones de evitarlo individualmente absteniéndose de actuar. La eventual abstención de cada uno de los agentes, individualmente considerados, no tiene capacidad alguna para detener el proceso que produce el efecto perverso. Y 
esto vale para todos los intervinientes, con independencia de su mayor o menor dotación de recursos. Así, por ejemplo, por fundamental que hayan sido las acciones del alcalde o de algún periodista en particular, su eventual abstención de esas acciones no hubiese tenido fuerza suficiente para detener el proceso, una vez puesto en marcha. Sería menester o bien una acción colectiva, o bien una acción individual deliberada y respaldada por recursos suficientes en cantidad y calidad.

El tercer rasgo particularmente perverso del caso consiste en lo que podría describirse como "progresión y autoaceleración del proceso». Las consecuencias no pueden concebirse linealmente como un momento $(C)$ respecto de un momento previo que sería el de la interacción $(I)$. Es más bien el resultado de un conjunto sucesivo de acciones e interacciones $(I, I, I \prime \ldots)$, cada una de las cuales da lugar a consecuencias $(C, C, C$ ”...) que, siendo "perversas» en sí mismas, se van reforzando y expandiendo progresivamente.

$$
I \rightarrow C \rightarrow I^{\prime} \rightarrow C^{\prime} \rightarrow I^{\prime \prime} \rightarrow C^{\prime \prime} \ldots
$$

Cada nueva «ronda» de interacción $\left(I, I^{\prime}, I^{\prime}\right)$ da nuevo impulso a la creencia en la existencia de una ola de crímenes $\left(C, C^{\prime}, C^{\prime}\right)$, en un proceso en autoexpansión que, a cada nuevo paso, exige para su detención una resistencia mayor.

La cuarta característica es que no se trata de un agregado de acciones casuales, fortuitas o en algún sentido fuera de lo común. Las acciones involucradas (las de los periodistas, los policías, los funcionarios y los públicos) están fuertemente institucionalizadas, y expresan pautas de acción reiterativas y relativamente estables. El efecto perverso se apoya en una constelación de intereses, procederes profesionales, costumbres y pautas, fijadas todas con relativa solidez, lo cual da al proceso buena parte de su fuerza y su estabilidad.

Lo cual nos lleva a la quinta característica, que es el carácter potencialmente contradictorio del proceso, en el siguiente sentido: aunque ninguno de los agentes que intervienen se propuso producir el resultado, cada uno de ellos, una vez puesto en marcha el proceso, actúa en forma por lo menos compatible con intereses inscriptos en su posición, de manera que revertir el proceso en marcha modificando la acción de los grupos intervinientes - los periodistas, los policías, los funcionarios y los públicos - implicaría algún tipo de obstáculo a la satisfacción de esos intereses. Así, por ejemplo, los periodistas tienen «motivos» para dejar que la ola de crímenes se alimente a sí misma, en la medida en que eso les proporciona cuotas de rating inusuales en momentos de "paz informativa». Y lo mismo vale para el personal policial: mientras dura la ola de crímenes, están en la palestra de la opinión pública, gozando de una atención inusitada sobre la labor que realizan y de una posibilidad poco común de hacer conocer sus demandas y, eventualmente, incrementar sus recursos. El proceso brinda al alcalde una atractiva posibilidad de satisfacer las demandas de la ciudadanía, en tanto los públicos tienen sus cuotas de satisfacción emotiva o psicológica. 
Está claro que estas características no tienen por qué serlo de todo caso de consecuencias involuntarias "perversas». Sin embargo, formalizan un tipo de fenómeno social al que le cabe con especial propiedad la expresión "perversidad». No sólo se trata de una resultante agregada «negativa» (es decir, un tipo particular de "consecuencia no intencionada"), sino también de unas propiedades del proceso que conduce a la resultante, de modo que ambas cosas (el proceso + su resultado) hacen del fenómeno un tipo muy particular de "hecho social». Tomando este caso como prototípico, podemos ensayar una formalización en los siguientes términos: se trata de la producción colectiva de un resultado indeseable, en la cual:

- ninguno de los participantes se propuso, a nivel de conciencia discursiva, producir el resultado;

- ninguno conoce, a nivel de conciencia discursiva, el resultado que se produce y los específicos mecanismos de su producción;

— todos los participantes tienen «razones» (a nivel de «conciencia discursiva», "conciencia práctica» o "motivaciones») para actuar como lo hacen;

- cada uno aporta, en proporción diversa según sus recursos, a la producción del resultado;

- ninguno puede, con el sólo expediente de abstenerse de actuar, impedir la producción del resultado;

- el resultado es opuesto a lo pretendido (a nivel de "conciencia práctica» o «discursiva») por al menos una parte de los participantes;

- el proceso no tiene la forma lineal y cerrada de un conjunto de acciones seguidas por un resultado; es el resultado progresivo de diversas acciones, cada una de las cuales ratifica y fortalece la dirección del proceso;

- las acciones de los participantes tienen un grado importante de fijeza e institucionalidad, por lo cual

- la evitación del efecto implica alterar, junto con las acciones, algunos de los intereses.

\section{A modo de conclusión}

Hay que insistir en que este ejercicio tiene sólo un carácter ilustrativo respecto de la tarea a realizar. Como la nueva noción ya no se basa en la simulación ideal típica de contextos de interacción, sus elementos sólo pueden formalizarse analizando la mayor cantidad posible de casos empíricos. Sin embargo, y con todo lo que tiene de provisoria, la formalización alcanza para mostrar no sólo la posibilidad de la tarea, sino también algo de su rentabilidad. Como muchos otros, el caso que analiza Fishman tiene una lógica, y hace intervenir unas variables, que resultan virtualmente invisibles para una teoría de la acción que no contemple, por ejemplo, la coexistencia de múltiples motivaciones, o la gravitación de la "conciencia práctica", la disponibilidad diferenciada de «recursos» o el «sentido». La indagación de esas lógicas y variables, y de los modos como actúan para producir resul- 
tados indeseables, es la tarea propia de una noción ampliada de «efectos perversos".

Por supuesto, esto implica asumir los costos del emprendimiento, que son principalmente metodológicos. Por formal que pueda resultar la definición, el análisis que ella permite (y al que obliga) no tiene más remedio que cargar con toda la opacidad de su objeto, alejándose por tanto de la pureza y formalidad que distingue a los modelos racionales. Max Weber describía al tipo ideal como «un puerto de refugio" para aprender a orientarnos en el «inmenso mar» de los hechos empíricos. Una noción ampliada de «efectos perversos» supone precisamente abandonar el puerto seguro de cualquier análisis formal, y adentrarse en el mundo inestable y complejo de los hechos empíricos. La apuesta consiste en ganar en riqueza y profundidad lo que se pierda en precisión y generalidad.

\section{Bibliografía}

AgUIAR, Fernando (comp.) (1991). Intereses individuales y acción colectiva. Madrid: Pablo Iglesias.

AXELROD, Robert (1986). La evolución de la cooperación. Madrid: Alianza.

BAERT, Patrik (1991). "Unintended consequences: a typology and examples». International Sociology, 6, 2.

BECKER, G. (1980). «El enfoque económico del comportamiento humano». Información comercial española, 557, enero.

BoudON, Raymond (1981). La lógica de lo social. Madrid: Rialp.

- (1982). The Unintended Consequences of social Action. Londres: Macmillan Press Ltd. Ed. original: Boudon, R. (1977). Effets pervers et ordre social. PUF: París.

- (1986). «Individual Action, Aggregation Effects and Social Change». En Theories of Social Change. Oxford: Polity Press.

ELIAS, Norbert (1982). Sociología fundamental. Barcelona: Gedisa.

ELTER, Jon (1996). Tuercas y tornillos. Barcelona: Gedisa.

Fishman, Mark (1979). La fabricación de la noticia. Tres Tiempos.

GidDENS, Anthony (1987). Las nuevas reglas del método sociológico. Buenos Aires: Amorrortu.

- (1995). La constitución de la sociedad. Buenos Aires: Amorrortu.

HAYEK, F. (1978). «The Results of Human Action but not of Human Design». En

New Studies in Philosophy, Politics, Economics and the History of Ideas. Londres:

Routledge and Kegan Paul.

Hirschman, Albert (1994). Retóricas de la intransigencia. México: FCE.

LAMO DE ESPINOSA, Emilio (1990). La sociedad reflexiva. Madrid: CIS.

MERTON, Robert (1980). «Las consecuencias imprevistas de la acción social». En Ambivalencia sociológica y otros ensayos. Madrid: Espasa Calpe.

- (1989). «Unanticipated Consequences and Kindred Sociological Ideas: A Personal Gloss». En MongardinI, C.; TABONI, S. L'opera di R.K. Merton e la sociologia contemporanea. EGIG (Edizioni Culturali Internazionali Genova).

Olson, Mancur (1992). La lógica de la acción colectiva. México: Limusa-Noriega. Poundstone, William (1995). El dilema del prisionero. Madrid: Alianza. 
RAMOS TORRE, Ramón (1993). «Una introducción a las paradojas en las ciencias sociales». En Rodríguez IbÁNEZ, J.; LAMO De EsPinOsA, E. Problemas de teoría social contemporánea. Madrid: CIS.

SCHELLING, Thomas (1971). «Dinamics models of segregation». Journal of Mathematical Sociology, vol. 4.

VERÓN, Eliseo (1993). La semiosis social. Barcelona: Gedisa. 